\title{
Cestrum chiangi (Solanaceae), una especie nueva de Guerrero y Oaxaca, México
}

\section{Cestrum chiangi (Solanaceae), a new species from Guerrero and Oaxaca, Mexico}

Universidad Michoacana de San Nicolás de Hidalgo, Facultad de Biología, Laboratorio de Sistemática Molecular de Plantas, Ciudad Universitaria, Av. Francisco J. Múgica s/n, 58030 Morelia, Michoacán, México.

Autor para la correspondencia: montero.umsnh@gmail.com

Recibido: 10 de abril de 2018.

Revisado: 9 de mayo de 2018.

Aceptado: 29 de mayo de 2018.

Primero en línea: 30 de mayo de 2018.

Publicado: 1 de julio de 2018.

Citar como:

Montero Castro, J. C. 2018. Cestrum chiangi (Solanaceae), una especie nueva de Guerrero y Oaxaca, México. Acta Botanica Mexicana 124: 219-224. DOI: 10.21829/abml24.2018.1376

DOI:

$10.21829 / a b m 124.2018 .1376$

\author{
Juan Carlos Montero Castro (i)
}

\section{RESUMEN:}

Antecedentes y Objetivos: Como otros géneros de plantas vasculares mexicanas, Cestrum necesita una revisión taxonómica. El género agrupa 36 especies para el país, pero seguramente faltan algunas por describir. Una de estas novedades taxonómicas es descrita aquí.

Métodos: Con base en material recolectado por el Dr. Alfonso Delgado Salinas, se realizó un análisis morfológico y una revisión bibliográfica, detectando una especie nueva para la ciencia.

Resultados clave: Se describe e ilustra Cestrum chiangi, especie endémica de la Sierra Madre del Sur, México. La flor de C. chiangi es morfológicamente similar a la de C. fulvescens.

Conclusiones: Esta especie es endémica de México y ocurre en localidades de Guerrero y Oaxaca.

Palabras clave: especie endémica, flora mexicana, novedad taxonómica.

\section{ABSTRACT:}

Background and Aims: Like other genera of Mexican vascular plants, more taxonomic work is needed in Cestrum. In Mexico, 36 species of the genus are distributed, but surely several more await to be described. One of these taxonomic novelties is described here.

Methods: Based on material collected by Dr. Alfonso Delgado Salinas, a morphological analysis and bibliographic review was realized, detecting a taxon new to science.

Key results: Cestrum chiangi, an endemic species of the Sierra Madre del Sur, Mexico, is described and illustrated. The flower of $C$. chiangi morphologically resembles to the flower of $C$. fulvescens.

Conclusions: Cestrum chiangi is endemic to Mexico and occurs in localities in Guerrero and Oaxaca. Key words: endemic species, Mexican flora, taxonomic novelty.

\section{INTRODUCCIÓN}

Entre los géneros de la familia Solanaceae, Cestrum L. es el segundo más diverso después de Solanum L. Agrupa de 150 a 175 especies (Benítez-de-Rojas y D’Arcy, 1998; Nee, 2001). Se ha estimado que en México hay 36 especies (Francey, 1935, 1936; Villaseñor, 2016); Martínez et al. (2017) reconocen 42, las cuales se distribuyen principalmente en la región sur del país. Entre las regiones y estados con más registros de especies de Cestrum se encuentran Chiapas (20) (Breedlove, 1986), Oaxaca (18) (Rodríguez, 2004), Veracruz (14) (Nee, 1986), Jalisco (11) (Martínez et al., 2017), Guerrero (9) (sumando reportes de diferentes estudios: Diego-Pérez et al., 2001; Martínez-Gordillo et al., 2004; Ávila-Sánchez et al., 2010; Cuevas-Guzmán 
y Montero-Castro, 2011; Montero-Castro, 2011), Valle de México (6) (Pérez-Hernández, 2001) y Michoacán (6) (Cué-Bär et al., 2006). En este estudio se describe una nueva especie distribuida en Guerrero y Oaxaca.

\section{Materiales y Métodos}

Se revisaron todas las especies de Cestrum reportadas para México, citadas en diferentes estudios (Hemsley, 1882; Standley, 1920; Nee, 1986; Pérez-Hernández, 2001; Villaseñor, 2016, Martínez et al., 2017), analizando las descripciones incluidas en la revisión del género (Francey, 1935; 1936). Además, la revisión incluyó especies de Centroamérica (Monro, 2012). Se revisaron ejemplares herborizados, incluyendo tipos, depositados en los herbarios CIMI, EBUM, ENCB, F, G, IBUG, IEB, MEXU, MO, QMEX y US. Para determinar el estado de conservación de esta especie, se aplicaron criterios de la IUCN (2017) relacionados con la dimensión del área de distribución.

\section{Resultados}

Cestrum chiangi Mont.-Castro, sp. nov. Fig. 1.

TIPO: MÉXICO. Guerrero, municipio Atlamajalcingo del Monte, $4 \mathrm{~km}$ al NW, sobre desviación Malinantepec - Tlacoapa, $2704 \mathrm{~m}$, bosque mesófilo de montaña, 28.V.2006, A. Delgado-Salinas 2553 (holotipo: EBUM!, isotipo: MEXU!).

Shrub tomentose, mostly on young vegetative parts, calyx and corolla lobes with dendroid trichomes; calyx campanulate with conspicuous ribs, calyx lobes acuminate, covering up to $3 / 4$ of the corolla tube and larger than the fruit, although it does not enclose it completely; corolla lobes folded longitudinally forming pyramidal structures.

Arbustos, 2-5 m de porte; ramas glabrescentes, pubescencia de tricomas dendroides en ramas jóvenes, aumentando conforme se aproxima al ápice; peciolo 2-5.2 cm de largo, pubérulo, lámina foliar ovada-lanceolada a elíptica, consistencia cartácea, ápice agudo a acuminado, base cuneada a redondeada, 8-12 pares de nervaduras laterales, haz pubérulo, con tricomas simples ligeramente recurvados, envés tomentoso con tricomas dendroides, 12.8-17.7 cm de largo, 4.2-8.2 cm de ancho, filotaxia alterna sin pseudoestípulas; cimas terminales y axilares, congestionadas, 12-36 flores, 8-12 cm de longitud, 5.5-9 $\mathrm{cm}$ de ancho, no se conoce que emitan aroma; bracteolas filiformes, caducas, 5-7 mm de largo, 1.5-2.5 mm de ancho; flores pentámeras, sin pedicelo; cáliz campanuliforme, laxo, color verde, $15-17 \mathrm{~mm}$ de largo, 4-4.5 mm de ancho, exterior tomentoso de tricomas dendroides, con costillas notables, lóbulos acuminados, 5-6 mm de largo, 1.2-1.6 mm de ancho; corola color verde amarillenta, hipocraterimorfa robusta, tubo externamente glabro, 19-21 $\mathrm{mm}$ de largo, primer tercio basal cilíndrico, $1.5-1.9 \mathrm{~mm}$ de diámetro, ampliándose gradualmente hasta 2.4-2.8 mm de diámetro en el sublimbo, donde se constriñe ligeramente, lóbulos ovados, externamente tomentosos, 5-6 mm de largo, $2 \mathrm{~mm}$ en la base, 4-4.6 $\mathrm{mm}$ en su ancho mayor, dobleces marginales $\pm 1 \mathrm{~mm}$ de ancho, permaneciendo plegados al inicio de la antesis, dando a los lóbulos un aspecto prismático; estambres con filamentos fusionados a la base del tubo de la corola en los primeros 10.9-12.7 mm, hasta ese punto pilosos con tricomas simples, continuándose con un ligero engrosamiento y una porción libre 4.6-5.7 $\mathrm{mm}$ de longitud, anteras $0.9-1.1 \mathrm{~mm}$ de longitud; ovario ovoide, 1.5-1.7 mm, óvulos 10-14, estilo 15.2-16.1 mm de largo; estigma capitado, $0.9 \mathrm{~mm}$; fruto una baya esferoidal, color blanco verdosa, 7-11 mm de diámetro; cáliz acrescente, sobrepasando el largo del fruto, sin cerrarse en la parte apical; semillas irregularmente trapezoidales, reticuladas, color negro, 3.5-4.6 mm de largo, $2 \mathrm{~mm}$ de ancho.

Hábitat y fenología: Cestrum chiangi se conoce de tres localidades de Guerrero y dos de Oaxaca. Crece en el bosque de pino-encino, entre 2335 y $2600 \mathrm{~m}$ de elevación. Se asocia con Abies Mill., Alnus Mill., Chiranthodendron Larreat. y Clethra L. Florece entre marzo y abril, y fructifica en julio. Sin embargo, como otras 


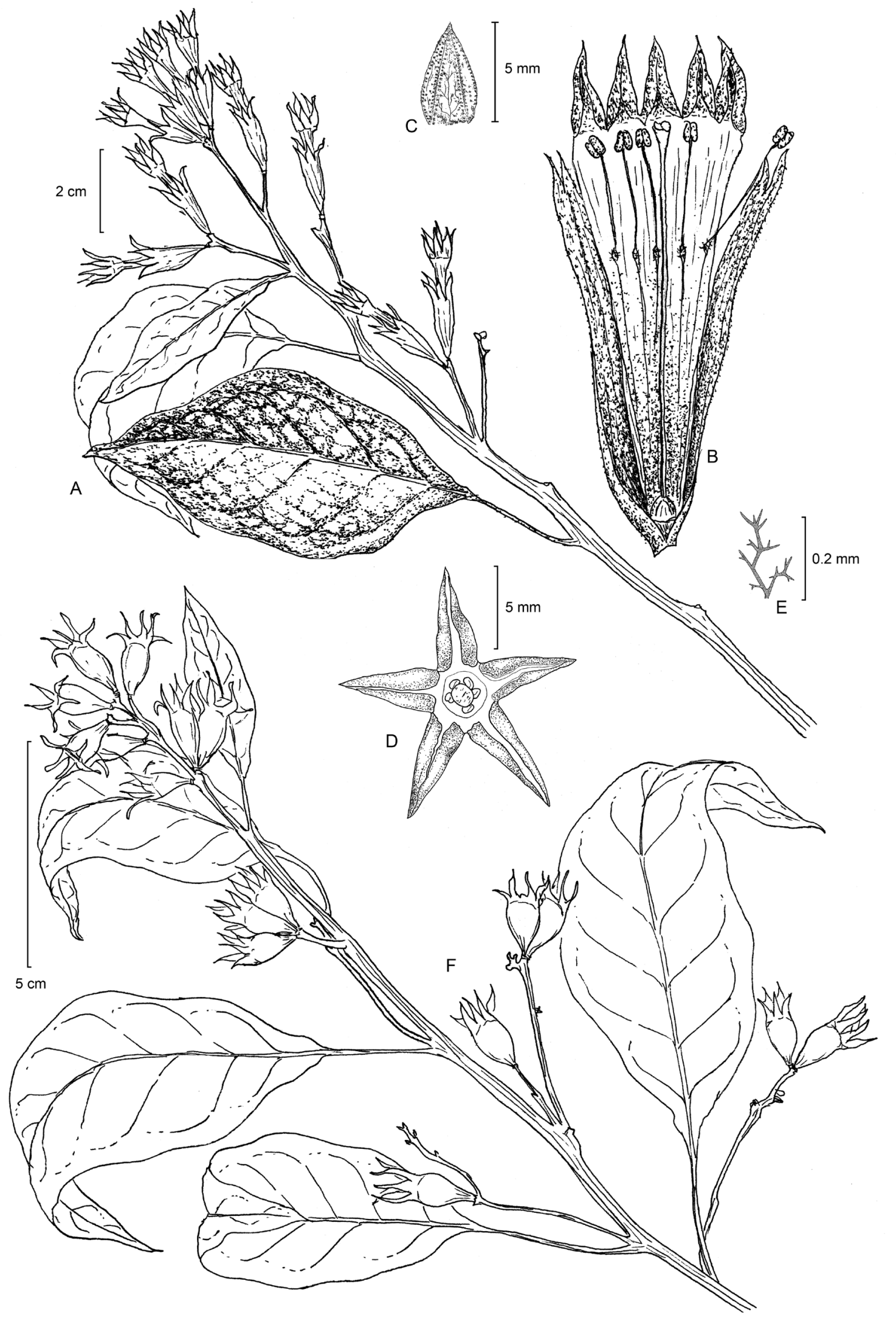

Figura 1: Cestrum chiangi Mont.-Castro (Delgado-Salinas 2553, holotipo: EBUM!) A. rama con flores; B. disección longitudinal de la flor; C. lóbulo de corola con pliegues laterales extendidos; D. vista apical del limbo de la flor; E. tricoma; F. rama con frutos envueltos en cáliz acrescente. 
especies de Cestrum, es probable que esta especie presente más de una temporada de floración.

Material adicional examinado: MÉXICO. Guerrero, municipio Chilchihualco, $5 \mathrm{~km}$ al W de Camotlán, $J$. Rzedowski 16385 (ENCB, MEXU); 8 km al SW de Filo de Caballo, J. C. Soto 8831 (MEXU). Oaxaca, municipio Santiago Juxtlahuca, a $3.5 \mathrm{~km}$ de la desviación de San Juan Piñas, J. I. Calzada 21759 (MEXU). Municipio Santiago Textitlán, El Chiquihuite, $R$. Trujillo 83 (MEXU).

Etimología: El epíteto específico honra al Dr. Fernando Chiang Cabrera, como un reconocimiento a sus aportes en el conocimiento de la familia Solanaceae en México y a sus exploraciones botánicas realizadas en el sur de México.

\section{DIscusión}

Cestrum chiangi desarrolla un cáliz de $15-17 \mathrm{~cm}$ y cubre $3 / 4$ de la corola, lo cual no es común en las especies mexi- canas del género. Sin embargo, se podría confundir con Cestrum fulvescens Fernald, aunque el cáliz de C. chiangi es tomentoso, con lóbulos acuminados y las costillas, aunque conspicuas, no son aladas (Fig. 2A), mientras que el cáliz de $C$. fulvescens es glabro con lóbulos deltoides irregulares y con costillas aladas (Fig. 2B). Otras especies en México que presentan cáliz tomentoso son C. fasciculatum (Schltdl.) Miers, C. roseum Kunt, C. mortonianum J.L. Gentry y C. tomentosum L. f. Las primeras dos especies no se pueden confundir, ya que presentan tricomas simples y el color de cáliz y corola es en tonos rojizos. Cestrum mortonianum, C. tomentosum y C. chiangi tienen tricomas dendroides y el color de cáliz y corola es verdoso, pero el cáliz excepcionalmente largo de $C$. chiangi sirve para discriminarla. Las primeras presentan un cáliz corto, menor a $1 / 3$ del tubo de corola y sin costillas evidentes.

De acuerdo a los criterios de la IUCN (2017), si se considera la extensión de ocurrencia de la especie (5430 $\mathrm{km}^{2}$ ), a $C$. chiangi le corresponde la categoría Vulnerable,

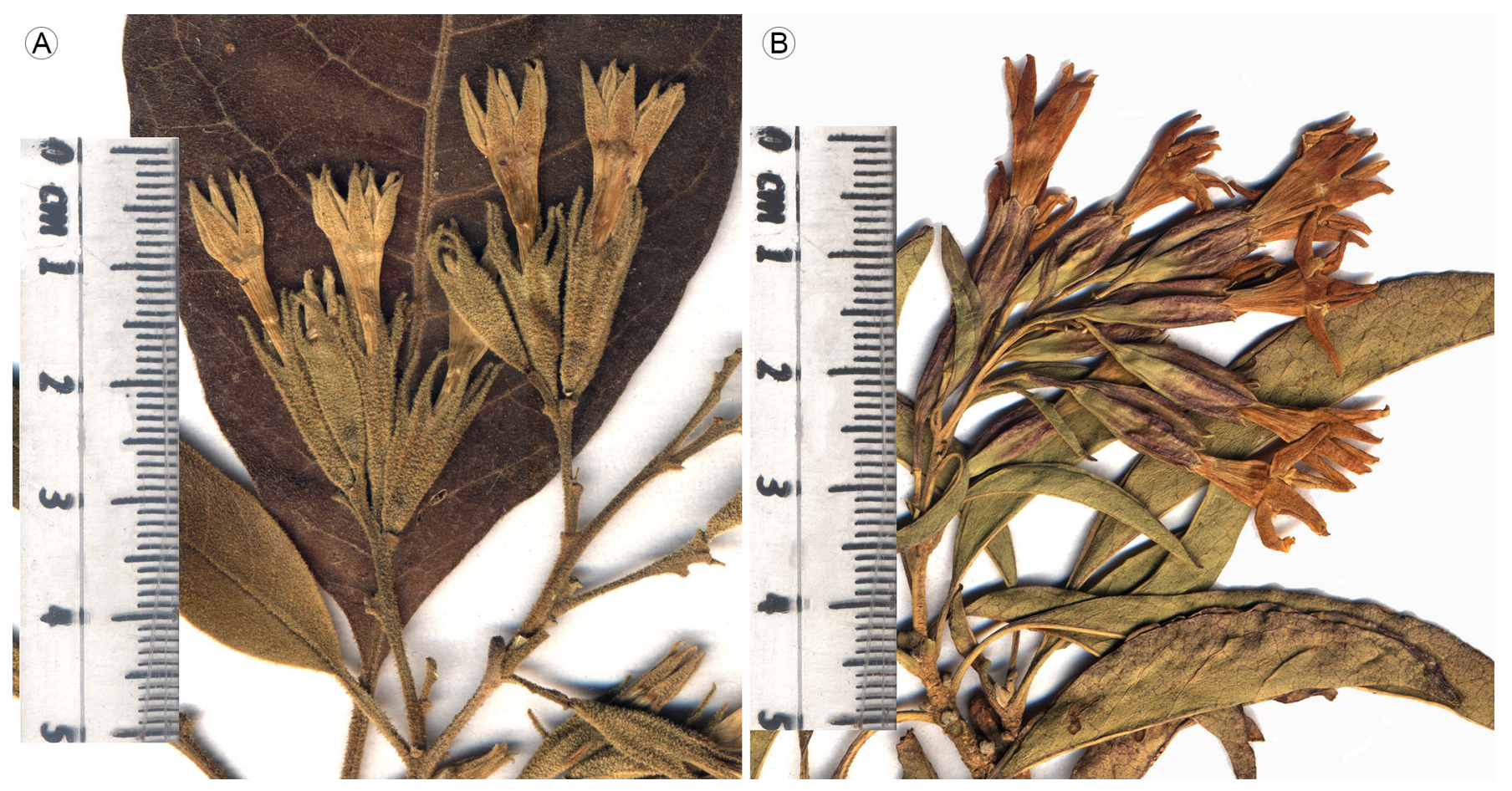

Figura 2: A. inflorescencia de Cestrum chiangi Mont.-Castro (Delgado-Salinas 2553, holotipo: EBUM!); B. inflorescencia de C. fulvescens Fernald (Medina 1562, EBUM). 
en cambio si se toma en cuenta el área de ocupación (20 $\mathrm{km}^{2}$ ) puede ser considerada En Peligro de Extinción. Para precisar el estado de conservación de esta especie será necesario evaluar en campo la condición de sus poblaciones.

\section{CONTRIBUCIÓN DE AUTOR}

JCMC ideó, realizó el estudio y es responsable por la redacción del documento. Así mismo, atendió las observaciones de los revisores, realizando las correcciones hasta la aprobación del manuscrito.

\section{FINANCIAMIENTO}

Esta investigación fue apoyada por la Coordinación de la Investigación Científica, Universidad Michoacana de San Nicolás de Hidalgo (UMSNH).

\section{AgradeCIMIENTOS}

Se agradece a Alfonso Delgado Salinas por las fotos y el material herborizado de sus colectas. A los curadores de las colecciones CIMI, EBUM, ENCB, F, G, IBUG, IEB, MEXU, MO, QMEX y US por su ayuda. Particularmente se agradecen los comentarios de los revisores anónimos que enriquecieron notablemente el documento. Esteban Martínez Salas buscó y encontró colectas de Cestrum entre el material herborizado y sin procesar del herbario MEXU, gracias. Carlos Ramírez Gaytán elaboró la ilustración.

\section{LITERATURA CITADA}

Ávila-Sánchez, P., A. Sánchez-González y C. CatalánEverástico. 2010. Estructura y composición de la vegetación del Cañón del Zopilote, Guerrero, México. Revista Chapingo, Serie Ciencias Forestales y del Ambiente 16(2): 119-138. DOI: https://doi.org/10.5154/r. rchscfa.2010.02.003

Benítez-de-Rojas, C. B. y W. G. D’Arcy. 1998. The genera Cestrum and Sessea (Solanaceae: Cestreae) in Venezuela. Annals of the Missouri Botanical Garden 85(2): 273-351. DOI: https://dx.doi.org/10.2307/2992010

Breedlove, D. E. 1986. Flora de Chiapas. Listados Florísticos de México IV. Instituto de Biología, Universidad Nacional Autónoma de México. Cd. Mx., México. 246 pp.
Cué-Bär, E. M., J. L. Villaseñor, L. Arredondo-Amezcua, G. Cornejo-Tenorio y G. Ibarra-Manríquez. 2006. The tree flora of Michoacan, Mexico. Boletín de la Sociedad Botánica de México 78: 47-81.

Cuevas-Guzmán, R. y J. C. Montero-Castro. 2011. Nuevo registro de Cestrum mortonianum (Solanaceae) y descripción de una variedad en la vertiente pacífica de México. Acta Botanica Mexicana 95: 37-43. DOI: https:// doi.org/10.21829/abm95.2011.265

Diego-Pérez, N., S. Peralta-Gómezy B. Ludlow-Wiechers. 2001.El Jilguero. Bosque mesófilo de montaña. Estudios Florísticos en Guerrero, No. 11. Prensas de Ciencias. Universidad Nacional Autónoma de México. Cd. Mx., México. 42 pp. Francey, P. 1935. Monographie du genre Cestrum L. Candollea 6: 46-398.

Francey, P. 1936. Monographie du genre Cestrum L., partie II. Candollea 7: 1-132.

Hemsley, W. B. 1882. Biologia Centrali-Americana; or Contributions to the Knowledge of the Fauna and Flora of Mexico and Central America, vol. II. R. H. Poret and Dulau \& Co. London, UK. 658 pp.

IUCN. 2017. Guidelines for Using the IUCN Red List Categories and Criteria. Version 13. Prepared by the Standards and Petitions Subcommittee. Downloadable from: http:// cmsdocs.s3.amazonaws.com/RedListGuidelines.pdf

Martínez-Gordillo, M., R. Cruz-Durán, J. F. Castrejón-Reyna, S. Valencia-Ávalos, J. Jiménez-Ramírez y C. A. RuizJiménez. 2004. Flora vascular de la porción guerrerense de la Sierra de Taxco, Guerrero, México. Anales del Instituto de Biología, Universidad Nacional Autónoma de México, Serie Botánica 75(2): 105-189.

Martínez, M., O. Vargas-Ponce, A. Rodríguez, F. Chiang y S. Ocegueda. 2017. Solanaceae family in Mexico. Botanical Sciences 95(1): 131-145. DOI: https://doi.org/10.17129/ botsci. 658

Monro, A. 2012. Eight new species of Cestrum (Solanaceae) from Mesoamerica. PhytoKeys 8: 49-82. DOI: https://doi. org/10.3897/phytokeys.8.2238

Montero-Castro, J. C. 2011. Cestrum sotonunezii, a new species of Solanaceae from central Mexico. Novon 21(2): 244248. DOI: https://doi.org/10.3417/2005040 
Nee, M. 1986. Solanaceae. In: Gómez-Pompa, A. (ed.). Flora de Veracruz 49. Instituto Nacional de Investigaciones sobre Recursos Bióticos. Xalapa, México. 191 pp.

Nee, M. 2001. An overview of Cestrum. In: Van den Berg, R. G., G. W. M. Barendse, G. M. Van der Weerden y C. Marinni (eds.). Solanaceae V: Advances in Taxonomy and Utilization. Nijmegen University Press. Nijmegen, Netherlands. Pp. 109-136.

Pérez-Hernández, S. 2001. Cestrum. In: Rzedowski, G. C. de y J. Rzedowski (eds.). Flora Fanerogámica del Valle de México. 2a ed., Instituto de Ecología, A.C. y Comisión Nacional para el Conocimiento y el Uso de la Biodiversidad. Pátzcuaro, México. Pp. 650-653.
Rodríguez, A. 2004. Solanáceas. In: García-Mendoza, A. J., M. de J. Ordóñez y M. Briones-Salas (eds.). Biodiversidad de Oaxaca. Fondo Oaxaqueño para la Conservación de la Naturaleza, World Wildlife Fund, Instituto de BiologíaUniversidad Nacional Autónoma de México. Cd. Mx., México. Pp. 297-300.

Standley, P. C. 1920. Trees and Shrubs of Mexico. Contributions from the United States National Herbarium 23(1): 12781283.

Villaseñor, J. L. 2016. Checklist of the native vascular plants of Mexico. Revista Mexicana de Biodiversidad 87(3): 559902. DOI: https://doi.org/10.1016/j.rmb.2016.06.017 